\section{Use of Melatonin in Children and Adolescents with Primary Sleep Disorders and Sleep Disorders Associated to Autism Spectrum Disorder and Attention Deficit-Hyperactivity}

\section{Abstract}

Background: Melatonin is an indoleamine often used for the treatment of primary and secondary insomnia in children and adolescents. Its hypnotic effect seems to be related to both, the reduction of the cortical activation, and the inhibition of the neural system responsible for the alertness. Goals of this study are to clarify the utility and safety of melatonin use in paediatric population and in adition to determine the dosage and the time of more effectiveness of melatonin administration in children and adolescents with primary sleep disorders and those associated with neurodevelopmental disorders.

Data Sources: A search was carried out on 'PubMed' with the keywords: "children insomnia melatonin"; "ADHD insomnia melatonin", "Autism spectrum disorder melatonin" restricted to publications of the last 10 years.

Results: The effectiveness of Melatonin in children and adolescent with Sleep Onset Delay (SOD) and with Delayed Sleep Phase Syndrome (DSPS) is consistently founded with different dosage, according to patient age and weight, and administered between 30 minutes and 3 hours before the apropiatte sleep onset. In children with ADHD the need for higher doses of melatonin has been set, specially in those taking medication for ADHD. In children with ASD lower doses and shorter intervals, from 30 to 60 minutes prior sleep onset has been suggested.

Conclusions: There is evidence of melatonin effectiveness in children and adolescents with sleep disorders, with few or no adverse effects and adequate security.

Keywords: Melatonin; Sleep disorders; Attention deficit hyperactivity disorder; Autism spectrum disorder; Children and adolescent

\section{Soraya Otero Cuesta and Ines Madrazo Delrio-Hortega \\ University Hospital Valdecila, Santander, Spain}

Corresponding Author:

Soraya Otero Cuesta

E sotero@humv.es

University Hospital Valdecila, Santander, Spain.

Citation: Cuesta SO, Delrio-Hortega IM. Use of Melatonin in Children and Adolescents with Primary Sleep Disorders and Sleep Disorders Associated to Autism Spectrum Disorder and Attention Deficit-Hyperactivity. J Pediatr Care. 2016, 1:1.

Received: November 26, 2015; Accepted: January 19, 2016; Published: January 27, 2016

\section{Introduction}

Melatonin is one of the most used substances for treating insomnia in children and adolescents. It is an indolamine synthesized in the pinealocytes of the pineal gland. Light plays an indispensable role in its action mechanism: photoperiodic information reaches the pineal gland through retinalhypothalamic pathway. Noradrenaline, released in this pathway via activation of $\beta 2$ receptors of the pineocyte induces expression of both enzymes ( $\mathrm{N}$-acetyltransferase (NAT) and hydroxyindole-Omethyltransferase (HIOMT) [1]. In the pinealocyte these enzymes synthesize melatonin from serotonin, previously synthesized from tryptophan. Due to the fact that natural light (also called blue or white ) inhibits this pathway, the melatonin synthesis reaches its maximum intensity between 2-3 am in normal conditions [2]. Melatonin has a concentration 20 times higher in CSF than in blood, has a half-life of 30 minutes and $70 \%$ circulates bound to albumin. It is metabolized in the liver by $95 \%$ and the remainder is eliminated intact thru the urine. Melatonin production is not rhythmic until 6 months of life. In fact, in premature infants there is an increased chance of developing disturbances in the sleepvigil rhythm [3]. This production is not stable throughout life: 
increases until puberty, when begins to decrease until a plateau period at 35-40 years. From 55-65 years, cells stop detecting light with the same sensitivity and thus begins a decrease of melatonin synthesis in old age, probably because the calcification of the pineal gland and the neuronal loss at the suprachiasmatic nucleus level.

Melatonin is mainly bound to three types of receptors, being the MT1 and MT2 the most important in sleep regulation. MT1, found in the suprachiasmatic nucleus, pars tuberalis and heart blood vessels, has chrono-biotic effects. MT2 are located in retina and hippocampus, being the type with greater affinity for melatonin [4].

At the end of the vigil period, when the increase in melatonin production begins, there is a decline in neurobehavioral activity that induces sleep, and this phenomenon is called the opening of sleep gate. Melatonin activation of GABA neurons in the reticular nucleus of the thalamus would be responsible for this reduction in cortical activity. Also a hypnotic effect of melatonin by its indirect action on GABA-BZD receptors is hypothesized. Besides the GABA neurons inhibit orexinergic neurons of the posterior lateral hypothalamus (neurons that promote wakefulness). The start of the vigil coincides with the decline of melatonin levels in blood [4]. Endogenous melatonin is involved in the circadian regulation of sleep and exogenous melatonin is able to modify this regulation [5].

Up to $40 \%$ of children with normal development present at some point difficulties initiating or maintaining sleep. This percentage rises to $80 \%$ in cases of children with the more prevalent neurodevelopmental disorders: Autism Spectrum Disorders (ASD) and Attention Deficit Disorder and Hyperactivity Disorder (ADHD), with negative impact on behavioral and cognitive domains [6].

The high frequency of sleep problems in chlidren justifies this review on the use of melatonin in their treatment., although the Spanish Agency for Medicines and Health Products does not authorize its use in pediatric insomnia [7].

In this review we will focus on treatment with melatonin for those primary sleep problems most prevalent in the population of this age: Sleep Onset Delay (SOD), Delayed Sleep Phase Syndrome (DSPS), and also for the sleep problems associated to ADHD and ASD. In adition our goals are to clarify the usefulness and safety of melatonin use in pediatric populations and to determine the dose and timing more appropriate for these patients.

\section{Methodology}

For the realization of this review a search was carried out on 'PubMed' with the following keywords: "children insomnia melatonin"; "ADHD insomnia melatonin", "Autism spectrum disorder melatonin" restricted to publications of the last 10 years in English or Spanish, extending this time if there were older references of special interest. Randomized clinical trials, reviews and meta-analysis are priorized, as are publications with peer review, and those articles focus on dose, timing of administration and effects of melatonin in the pediatric population. A few more additional papers with special theorical interest were considered too.

\section{Results}

Prior to the presentation of results of the review, we considered useful to specify the parameters and measurement methods used in the different studies.

Subsequently the clinical research data supporting the utility of melatonin for the treatment of primary insomnia and sleep problems associated to ADHD and ASD are presented, and precautions and limitations of its use in these populations are described.

\section{Glossary of parameters of sleep}

When studying the effectiveness of melatonin in the different types of insomnia in children and adolescents the following sleep parameters are used.

-Dim Light Melatonin Onset (DLMO): Is the moment when melatonin reaches a concentration in saliva higher than $4 \mathrm{pg} /$ $\mathrm{ml}$. DLMO it is considered the best marker of the circadian clock phase and it is set that preceded in two hours the onset of sleep, and is determined by analysis of melatonin of blood or saliva samples taken every 30-60 between $7 \mathrm{pm}$ and $11 \mathrm{pm}[2,8]$.

\section{Actigraphy}

Method used to determine the time parameters and sleep intervals, consisting of a sensor, usually placed in the wrist, which detects motor activity in children [8,9]. In actigraphy the following measures are used

a. Sleep onset $(\mathrm{SO})$ is the time of the sleep conciliation.

b. Sleep latency $(\mathrm{SL})$ is the interval between bedtime and SO. In most of the studies the interval is of 30 minutes while in others is set in 60 minutes.

c. Total sleep duration: is the interval between $\mathrm{SO}$ and awakening.

d. INT: interval between the administration of melatonin and DLMO. This measure is show to be quite useful for determining the time in which the administration of melatonin would be more effective.

\section{Use of melatonin in sleep onset disorder (SOD)}

The SOD is one of the most common sleep problems in children and adolescents. It is defined as falling asleep later than the socially accepted as valid for children up to 6 years. This time is set based on the average bedtime obtained in nationwide data for children this age. From the age of 6 , the reference time is obtained by adding 15 minutes more for each year older. For the diagnosis of SOD is necessary a sleep latency greater than 30 minutes, and presented in more than 4 nights per week [6]. The therapeutic indication of first line is Behavioural Therapy, and when is not effective the prescription of melatonin should be considered.

Administration of exogenous melatonin in children with insomnia generates a change in both DLMO and SO. The magnitude of these changes depends more of the time of administration than of dosage of melatonin. Analyzing the curve dose/time, the most significant effects of melatonin on SO occur when this is given 5 or 6 hours prior to baseline DLMO [10]. It is important to highlight that in children with SOD, DLMO is delayed an average of 50 minutes compared with normal children [6].

In a review by Kristiaan [6], it was found that when the DLMO basal was set later than 19:30 the administration of melatonin induced and advance of sleep conciliation of 25 minutes for 
each hour of delay of DLMO. In this review the DLMO prior to initiation of therapy is a good predictor of the best moment for administration of melatonin in these children.

Regarding the optimal dose, in several studies [6,8] and in a randomized controlled study [10], melatonin was demonstrated to be effective in children aged 6-12 years in a dose of $0.05 \mathrm{mg} /$ $\mathrm{kg}$ administered at least 1-2 hours before DLMO. It has also been proven to be effective if administered 30-60 minutes before bedtime [11].

\section{Use of melatonin in delayed sleep phase disorder (DSPD)}

The DSPD consists of a Primary Sleep Disorder in which there is a delay of almost two hours in the sleep conciliation but the total amount of sleep is normal. It is associated with polymorphisms in certain genes involved in circadian mechanisms. Excessive exposure to sun light, television, or video games, in the evening, or lack of exposure to sunlight during the day can exacerbate this problem [3]. The chronobiotic effect of melatonin on the MT2 receptors in the suprachiasmatic nucleus would be the responsible for the change in the momentum phase [12]. Based on the previously mentioned studies $[6,8]$ there is evidence of a change in timing phase following administration of exogenous melatonin. In this case it will depend solely on the time of administration, being more effective when it is situated about 5-6 hours before the basal DLMO. Regarding the dosage, the variability is great, between 0.5 and $5 \mathrm{mg}$, and according to age, between 2 to $3 \mathrm{mg}$ for children and between 5 to $10 \mathrm{mg}$ for adolescents $[2,8]$.

It is Important to highlight that these children and adolescents often tend to have irritability, concentration problems and difficulties in the cognitive domain as a result of problems arising from its sleep phase delay. These kids are likely to have problems in adaptation to the social rhythms of sleep-vigil, i.e waking up early to attend school activities and go to bed early. Interestingly some studies suggest that when they are treated with melatonin these problems also improve [8].

\section{Melatonin usage in children with ADHD}

Attention Deficit Hyperactivity Disorder (ADHD) is now considered a neurodevelopmental disorder, as it is included in DSM-5 [13]. It has a worldwide prevalence of between 5 and $12 \%$ in schoolage [14], with a high prevalence of commorbidities (59-87\%) with other conduct disorders, language, social adaptation problems, obesity and/or sleep problems. The interrelationship between $\mathrm{ADHD}$ and sleep problems is complex, possibly multidirectional and multifactorial, with a significant impact on the child's functioning during the day.

Sleep problems in those kids may be related to several factors: resistance in going to bed, lack of boundaries, increased nighttime restlessness, medication, and/or comorbidity associated with delayed sleep phase.

\section{SOD comorbid with ADHD}

A 33\% of children with ADHD without stimulant medication suffers $\mathrm{SOD}$, and the administration of melatonin decreased sleep latency and increased sleep duration. The doses that have been found effective are 3-6 mg for children between 6 and 12 years $[8,15]$. () Under License of Creative Commons Attribution 3.0 License
Some of the possible mechanisms in the genesis of sleep problems in these children are inherent to ADHD symptoms: behavioral disorders with problems in limits compliance, hyperactivity etc. In a small proportion of these cases an improvement in their sleep disorders when treated with specifics treatment for ADHD can be founded [16]. These sleep problems are associated with increased anxiety, impulsivity, inattention, and irritability during the day. It is likely that achieving an improvement in these parameters would improve sleep. However, the clinical research studies are not consistent in set a statistically significant relationship between sleep enhancement and improvemebt in behaviour and/or quality of life [8].

\section{Side effects in sleep due to pharmacologic treatments}

More often pharmacologic treatment approved for use in ADHD: methylphenidate in different presentations, Lisdexamfetamine and Atomoxetine can produce insominia of conciliation or significantly aggravate a previous sleep disorder. There are several studies in which the effectiveness of melatonin is evaluated in samples of children and adolescents diagnosed with ADHD and treated with stimulants [15]. There is a consistent evidence of a decrease in sleep latency after administration of doses of between 5 and $10 \mathrm{mg}$ of melatonin, taken between 20 minutes and two hours before going to bed $[8,17]$.

\section{Use of melatonin in children with autism spectrum disorder}

This is one of the areas in which the effectiveness of melatonin has been most studied. There are tudies $[17,18]$ that show a prevalence of between 40 and $86 \%$ of sleep problems in children with ASD, including conciliation problems, maintenance and decreased of total sleep duration. These problems also correlate with changes in mood, gastrointestinal problems, and greater difficulties in social interaction of these children, as well as an increase of stereotypies and communication problems [9].

Alterations in the secretion of melatonin may play a role in the development of symptoms in these children with ASD. In fact, alterations in genes involved in circadian rhythms have been found, linking autism with a deficit in the HIOMT enzyme [8]. Moreover, there is additional evidence for the association of problems in melatonin secretion with sleep problems in children with ASD, although these are mainly attributed to complex interactions between biological, psychological, social and environmental factors $[8,19]$.

Malow analyzed the relationship between different doses of melatonin according to age and weight. The sample consisted of 24 children between 3 and 10 years with ASD [18]. They all showed an increase in sleep latency more than $\mathbf{3 0}$ minutes. It was observed that there was no need of a higher dose than $6 \mathrm{mg}$ to improve the sleep parameters being $3 \mathrm{mg}$ the dose that achieved better response.

In a systematic review by Rossignol and Frye [9], 7\% of children with ASD and sleep problems take melatonin for treatment. The findings refer to several studies reflecting improved behavioral disturbances in children treated with melatonin at night. Also better measures in several sleep parameters as are total sleep 
duration, sleep quality, latency and sleep maintenance are evident, although the data of the various studies are not consistent regarding paarmeters of sleep maintenance. The authors suggest that these differences might be due to the use of sustained modified release melatonin in those studies finding a positive effect of melatonin in sleep maintenance in kids with ASD. The recommended dose ranges between 0.75 and $15 \mathrm{mg}$, in subejts between 2 and 18 years, with a great variability in treatment duration (between 14 days and four years) in different samples. Based on these findings we suggest that sustained-release melatonin would be helpful in improving the overall duration, maintenance and quality of sleep in children with ASD, while would have no effect on sleep onset. In those cases with ASD and SOD inmediate release melatonin would be indicated. According to these results, the use of both types of melatonin: immediate release and modified-release in those children and adolescents with ASD who present alterations in multiple parameters of sleep, could be suggeted, and would be a hypothesis to test for new coming research about this topic.

In a randomized placebo-controlled study the difference in efficacy in the treatment of insomnia in children with ASD between 4 and 10 years between melatonin ( $3 \mathrm{mg}$ at 21:00) and cognitive behavioral therapy was analyzed and melatonin was most effective in the treatment of insomnia that CBT alone. According to other studies, the use of both treatments generated better results than each one separately [19].

\section{Other}

There are also references to the use of melatonin in other sleep problems, and parasomnias or restless legs syndrome (RLS), for which there is no consistent evidence of its effectiveness [2], Other properties assigned to melatonin would be its antioxidant effect but more evidenec is needed [20].

\section{Side effects in melatonin}

The use of melatonin in the pediatric population with sleep problems is high, often without a medical prescription. The ease of use and lack of side effects is well documented.

Most studies do not find symptoms of drowsiness, neither development of dependence nor discontinuation syndrome, which are advantages comparing to benzodiazepines. Development of tolerance during the administration of melatonin is not described either, although there is some evidence of tolerance when using melatoninergic agonists in children with neurological problems, probably due to alterations in metabolism that are still to be studied [4].

Compared with placebo there is not evidence of increase in side effects, as headache, nausea, abdominal discomfort, mood disturbances, dizziness, etc. in short-term studies [8]. Some rare side effects as are night flushing, cold feeling and dizziness have been found in one study, the frequency of occurrence being directly related to dosage: the higher the dose the more prevalence of side effects [10]. There are not studies analyzing the effects of melatonin use in long term, but in one study conducted during three years no adverse effects regarding sleep quality, puberal development or health mental parameters were evident in children to whom melatonin was administered continuously [21].

The possibility of a higher prevalence of adverse effects in children with neurological problems remains controversial. In one study was observed an increased frequency of seizures in 4 out of the 6 children with brain injuries [22]. Subsequent studies do not replicate these findings, remaining as inconclusive results. Even other studies attributed to melatonin anticonvulsant effects in children diagnosed with epilepsy $[23,24]$. Because of the lack of consistency in the findings, its use in children with epilepsy should be cautious, although there is no clear evidence of increase in seizures.

Several studies have ruled out the influence of melatonin on the reproductive system (by influence on the pituitary-gonadal axis) at young ages, and the EMA's (European Medicines Agency) contraindicated its use in children, adolescents and pregnant women [4], and does not approve the prescription of extendedrelease melatonin below 55 years. Possible immunostimulating effects of melatonin are also suggested, and consequently some precaution must be taken when used in patients with autoimmune diseases [4].

\section{Limitations of this review}

This review has some limitations regarding both, the availability of studies and the heterogeneity of the samples used in them. Most of the articles found in the literature are mixed samples of children and adults, moreover the samples are not homogeneous in terms of age and clinical profiles and some of them are too small to get valid conclusions. These facts make difficult to clarify indications for our patients and to set consistent data on appropriate dose and time for each specific disorder.

\section{Conclusions}

1. The use of melatonin for treating insomnia in the pediatric population is widespread, and taking into account the findings, is effective in most cases. There is sufficient evidence of its positive effect in SOD in both healthy children and in children with neurodevelopmental disorders.

2. In general it is recommended the use of melatonin in doses from 0.5 to $10 \mathrm{mg}$ administered 60 minutes before bedtime, more specifically:

In children with SOD a dose of $0.5 \mathrm{mg} / \mathrm{kg}$ administered between 1 and 2 hours before bedtime is recommended.

a. In children with DSPD is recommended a dosage between 2-3 $\mathrm{mg}$ for children and 5-10 mg for adolescents administered 5 to 6 hours before DLMO. The availability of sleep measures as DLMO prior of treatment with melatonin is highly recommended. This data allow establishing the adequate timing for each patient in which there was greater likelihood of effectiveness. If it is not possible to determine the DLMO, melatonin taken 3 hours prior to going to bed is recommended.

b. In children with ADHD and associated sleep problems:

c. In case of not being medicated with stimulants, doses between 3 and $6 \mathrm{mg}$ given 30-60 minutes before bedtime are recommended.

d. In case of treatment with methylphenidate, effective doses 
are higher, between 5 and $10 \mathrm{mg}$ of melatonin administered between 30-60minutes before bedtime.

e. In children with ASD and sleep problems a dose of 3 to $6 \mathrm{mg}$ is recommended. administered at least 30 minutes before going to bed.
3. Because of its safety and few side effects, effective doses can be prescribed in most children, taking special care in children with epilepsy, inflammatory diseases or tumors for their immunestimulatory properties and possible epileptic effect. 


\section{References}

1 Wurtman R (2015) Physiology and available preparations of melatonin.

2 Pin Arboledas G, Merino Andreu M, de la Calle Cabrera T, Hidalgo Vicario MI, Rodríguez Hernández PJ, et al. (2014) Consensus on the use of melatonin in children and adolescents with difficulties initiating sleep. An Pediatr 81: 328.e1-9.

3 Escames G, Acuna-Castroviejo D (2009) Melatonina, análogos sintéticos y el ritmo sueño/vigilia. Rev Neurol 48: 245-254.

4 Hardeland R (2009) New approaches in the management of insomnia: weighing the advantages of prolonged-release melatonin and synthetic melatoninergic agonists. Neuropsychiatr Dis Treat 5: 341-354.

5 Arendt J, Skene DJ, Middleton B, Lockley SW, Deacon S (1997) Efficacy of melatonin treatment in jet lag, shiftwork, and blindness. J Biol Rhythms 12: 604-617.

6 Van der Heijden KB, Smits MG, Van Someren EJW, Boudewijn Gunning W (2005) Prediction of melatonin efficacy by pretreatment dim light melatonin onset in children with idiopathic chronic sleep onset insomnia. J Sleep Res 14: 187-194.

7 Spanish Health National System (2012) Clinical practice guideline. Trastornos del Sueño en la Infancia y la Adolescencia en Atención Primaria.

8 Sánchez-Barceló EJ, Mediavilla MD, Reiter RJ (2011) Clinical Uses of Melatonin in Pediatrics. Int J Pediatr 2011: 1-11.

9 Rossignol DA, Frye RE (2011) Melatonin in autism spectrum disorders: a systematic review and meta-analysis. Dev Med Child Neurol 53: 783-792.

10 Van Geijlswijk IM, Van der Heijden KB, Egberts ACG, Korzilius HPLM, Smits MG (2010) Dose finding of melatonin for chronic idiopathic childhood sleep onset insomnia: an RCT. Psychopharmacology (Berl) 212: 379-391.

11 Gitto E, Aversa S, Reiter RJ, Barberi I, Pellegrino S (2010) Update on the use of melatonin in pediatrics. J Pineal Res 50: 21-28.

12 Owens JA (2009) Pharmacotherapy of Pediatric Insomnia. J Am Acad Child Adolesc Psychiatr 48: 99-107
13 (2014) Diagnostical and Stadistical Manual of Mental Disorsers, (5thedn). American Psychiatric Association.

14 Spruyt K, Gozal D (2011) Sleep disturbances in children with attention-deficit/hyperactivity disorder. Expert Rev Neurother 11: 565-577.

15 Cummings $C$ (2012) Melatonin for the management of sleep disorders in children and adolescents. Paediatr Child Health 17: 331-333.

16 Efron D, Lycett K, Sciberras E (2014) Use of sleep medication in children with ADHD. Sleep Med 15: 472-475.

17 Mohammad MD, Mostafavi SA, Keshavarz SA, Eshraghian MR, Hosseinzadeh P, et al. (2012) Melatonin effects in Methylphenidate treated children with attention deficit hyperactivity disorder: a randomized double blind clinical trial. Iran J Psychiatry 7: 87-92.

18 Malow B, Adkins KW, McGrew SG, Wang L, Goldman SE, et al. (2012) Melatonin for sleep in children with autism: a controlled trial examining dose, tolerability, and outcomes. J Autism Dev Disord 42: 1729-1737.

19 Cortesi F, Giannotti F, Sebastiani T, Panunzi S, Valente D (2012) Controlled-release melatonin, singly and combined with cognitive behavioral therapy, for persistent insomnia in children with autism spectrum disorders: a randomized placebo-controlled trial. J Sleep Res 21: 700-709.

20 Lin L, Huang Q, Yang S, Chu J, Wang J, et al. (2013) Melatonin in Alzheimer's Disease. Int J Mol Sci 14: 14575-14593.

21 Van Geijlswijk, Mol RH, Egberts TC, Smits MG (2011) Evaluation of sleep, puberty and mental health in children with long-term melatonin treatment for chronic idiopathic childhood sleep onset insomnia. Psychopharmacology (Berl) 216: 111-120.

22 Sheldon SH (1998) Proconvulsant effects of oral melatonin in neurologically disable children. Lancet 351: 1254.

23 Goldberg-Stern H, Oren H, Peled N, Garty BZ (2012) Effect of melatonin on seizure frequency in intractable epilepsy: a pilot study. J Child Neurol 27: 1524-1528.

24 Peled N, Shorer Z, Peled E, Pillar G (2001) Melatonin effect on seizures in children with severe neurologic deficit disorders. Epilepsia 42: 1208-1210. 